\title{
Über die durch Röntgenstrahlen erregte Fluoreszenz des Quecksilberdampfes.
}

\author{
Von J. Steph. van der Lingen in Kapstadt.
}

(Fingegangen am 27. April 1922.)

Im Jahre 1913 untersuchten Landau und Piwnikiewics das durch Röntgenstrahlen erregte Fluoreszenzspektrum des Quecksilberdampfes und kamen dabei zu dem Schluß, daß das Emissionsspektrum aus einer kontinuierlichen Bande besteht, die von $3300 \AA$ bis zum sichtbaren Spektrum reicht und ihre größte Intensität bei $4100 \AA$ hat ${ }^{1}$ ). Sie fanden ferner, daß die Intensität des Fluoreszenzlichtes mit der Dampfdichte wächst, daß aber dies Anwachsen nicht der Dampftemperatur proportional ist.

In einer kürzlich erschienenen Veröffentlichung wurde gezeigt, daß dieser Dampf, wenn er mit ultraviolettem Licht von Wellenlängen zwischen 1860 and $2550 \AA$ bestrahlt wird, im rubenden Zustande nicht erregt wird, daß vielmehr sichtbare Fluoreszenz nur bei der Destillation des Dampfes auftritt; dadurch wird bewiesen, daB die wirksamen Molekïle keine neutralen einatomigen Moleküle sind. Es wurde ferner nachgewiesen, daß die relative Intensität der Emissionsbanden sich sowohl mit der Wellenlänge der erregenden Linien als auch mit der Dampfdichte ändert ${ }^{2}$ ); ferner zeigte Wood im Jahre 1909, daß dieser Dampf im Vakuum die Linie $2536 \AA \AA$ emittiert, wenn er durch Aluminiumlinien, die bei $1860 \AA$ liegen, erregt wird, und daß die Intensität dieser Emissionslinie abnimmt, wenn die Dampfdichte eine gewisse Grenze überschreitet, so daß sie bei hoher Dampfdichte nicht mehr im Fluoreszenzspektrum auftritt ${ }^{3}$ ). Es ist möglich, daß früberen Beobachtern die Emissionslinien entgangen sind, weil sie ihre Untersuchangen bei einer zu hohen Dampfdichte anstelltev.

Die im folgenden beschriebenen Experimente sollen entscheiden, ob das durch Röntgenstrahlen erregte Fluoreszenzspektrum dem Spektrum des durch ultraviolettes Licht zwischen 1860 und $2550 \AA$ erregten Dampfes entspricht, und ob die Härte der Röntgenstrahlen irgend einen Einfluß auf die Natur des Emissionsspektrums ausübt.

Die benutzte Apparatur bestand aus einem kleinen evakuierten Quarzkolben, der ein Tröpfchen Quecksilber enthielt, einem Hilger-

1) St. Landau und H. Piwnikiewics, Phys. ZS. 14, 381-386, 1913.

2) J. Steph. van der Lingen und R. W. Wood, Astrophys. Journ. 54, $149-160,1921$.

3) R. W. Wood, Phil. Mag. (6) 18, 187-193, 1909. 
schen Quarzspektrographen, einer Induktionsspule, die mit einem Wehneltschen Unterbrecher betrieben wurde, und einer mit Platinzuführungen versehenon Röntgenröhre. Die Röntgenröhre befand sich in einem mit Blei ausgekleideten Kasten, in dem ein kleines Loch in der Höhe des Spektrographenschlitzes angebracht war; das Quarzkölbchen war mit einem Stückchen Kupferdraht ror dem Loch aufgehängt, so daß die Röntgenstrahlen den zentralen Teil desselben durchsetzten. Das Quarzkölbchen wurde durch einen Bunsenbrenner erhitzt, der in einem Asbestrohr aufgestellt war, und der Spektrograph wurde auf die Seite des Kölbchens fokussiert, auf welcher die Röntgenstrahlen eintraten; dies geschah, um eine mögliche Absorption des Fluoreszenzlichtes durch den Dampf selbst zu vermeiden. Die Öfnung im Bleikasten' war mit einem Stückchen schwarzen Papiers bedeckt, um einen guten Hintergrund für die Untersuchung des Fluoreszenzlichtes zu schaffen, und auch, um das von der Röntgenröhre ansgehende Fluoreszenzlicht, welches etwa durch Reflexion an dem Quarzkölbchen in den Kollimator des Spektrographen gelangen könnte, unschädlich zu machen. - Zum Vergleich war eine Cadmium-Funken. strecke in der Nähe des Quarzkölbchens aufgestellt.

Die Methode der Untersuchung bestand darin, daß man das Kölbchen stufenweise erwärmte und beobachtete, bei welcher Temperatur sichtbare Fluoreszenz auftrat, wenn der Dampf durch den Cadmiamfunken erregt wurde, und dann auf die Röntgenstrahlen umschaltete. Durch abwechselnde Betätigung des Funkens und der Röntgenstrablen fand man, daß die sichtbare Fluoreszenz ungefäbr bei derselben Temperatur erschien, aber daß die Intensität größer war im Falle des Funkens. Bei steigender Dampfdichte wuchs die Intensität der sichtbaren Fluoreszenz in beiden Fällen, jedoch mit dem Unterschied, daß der Fluoreszenzstrahl im Falle der Funkenerregung bis auf die Fläche des Kölbchens zurückwich, während er bei Röntgenstrahlerregung immer die ganze Dicke des Kölbchens durchdrang. Dies würde man erwarten können, wenn die Röntgenstrablen nicht so leicht im Dampf absorbiert würden, wie das altraviolette Licht des Funkens. Rohe Messungen mit einem Elektroskop zeigten, daß die Absorption der Röntgenstrahlen sehr klein ist.

Bei Beobachtung der Oberfläche des Kölbchens zunächst der Röntgenröhre wurde gefunden, daß das Kölbchen selbst nicht mit sichtbarem Licht fluoresziert, wie es es tun würde; wenn es durch Strahlen von der Wellenlänge $2300 \AA$ ̊ getroffen würde. (Die Cadmiumlinien in der Gegend von 2266 bis $2350 \AA$ geben eine helle Fluoreszenz, die bis $3100 \AA$ reicht und etwa bei $3500 \AA$ ihr Maximum besitzt.) 
40 J. Steph. van der Lingen, Über die durch Röntgenstrahlen erregte Fluoreszenz.

Das Flnoreszenzspektrum des Dampfes von kleiner Dichte, das durch weiche, d. h. durch solche Röntgenstrahlen erregt ist, welche durch eine $1 \mathrm{~mm}$ dicke Glasplatte absorbiert werden, besteht, wie durch die Photographie gefunden warde, aus zwei strukturlosen Banden und fünf Emissionslinien. Die Banden reichen von 4350 bis 5500 und von 3100 bis $3600 \AA$ und haben ihre Maxima bei 4350 nnd bei $3300 \AA$ A. Die Emissionslinien sind $3140 \AA, 3660 \AA, 4050 \AA$, $4350 \AA$ und $5500 \AA$; sie konnten nicht sämtlich auf einer und derselben Platte erhalten werden. Die Linie $5500 \AA$ ist sehr schwach und kann nur schwer herausentwickelt werden; sie wird sehr leicht durch die Bande verdeckt. Die Linien $3140 \AA$ und $3660 \AA$ sind gleichfalls schwach und können nur erkannt werden, wenn auch die ultraviolette Bande schwach ist. Die Linien $4050 \AA$ and $4350 \AA$ sind hell und kräftig und verschwinden nur, wenn die Dampfdichte wächst; $4350 \AA$ ist kräftiger als $4050 \AA$.

Wiederholte man diese Versuche mit härteren Röntgenstrahlen und hielt dabei die Dampfdichte niedrig, so erschienen die Linien $4050 \AA$ und $4350 \AA$ auf der Platte, ebenso auch die Bande oberhalb $4350 \AA$ A. Dagegen war die ultraviolette Bande sehr schwach und von $4050 \AA$ nach dem ultravioletten Ende des Spektrums zu allmählich abschattiert; ohne ein Maximum bei $3300 \AA ̊ \AA$ zu zeigen. Bei höheren Dampfdichten verschwanden diese Iinien.

Die vorstehend geschilderten Versuche wurden mehrere Male wiederholt, aber in keinem Falle konnte irgend eine Spur der Linie $2536 \AA$ oder der Linie $2346 \AA$ gefunden werden, die zuerst von Steubing 1) entdeckt worden ist; die strukturlosen Banden bei $2346 \AA$ und $2536 \AA$ waren gleichfalls nicht vorhanden. Mit der möglichen Ausnahme der Bande im sichtbaren Spektrum bestebt keine Âhnlichkeit zwischen diesen durch Röntgenstrahlen und durch ultraviolettes Licht von den Wellenlängen zwischen 1860 und $2550 \AA$ erregten Fluoreszenzspektren.

Die Fluoreszenz dieses Dampfes, die auf verschiedenen Wegen erregt wird, soll in der nächsten Zeit eingehender untersucht werden.

Annliche Beobachtungen wurden mit Cadmiumdampf ausgeführt, aber es konnte weder eine sichtbare noch eine vltraviolette Flnoreszenz erhalten werden.

Baltimore, 24. November 1921.

1) W. Steubing, Phys. Z8. 10, 787, 1909. 